\title{
Usefulness of valacyclovir prophylaxis for cytomegalovirus infection after anti-thymocyte globulin as rejection therapy
}

\author{
Eun Jeong Ko, Ji Hyun Yu, Chul Woo Yang, and Byung Ha Chung
}

Transplant Research Center and Division of Nephrology, Department of Internal Medicine, College of Medicine, Seoul St. Mary's Hospital, The Catholic University of Korea, Seoul, Korea

Received: January 24, 2017

Revised : March 9, 2017

Accepted: March 15, 2017

\section{Correspondence to}

\section{Byung Ha Chung, M.D.}

Department of Internal

Medicine, College of Medicine,

Seoul St. Mary's Hospital,

The Catholic University of Korea, 222 Banpo-daero, Seocho-gu,

Seoul 06591, Korea

Tel: +82-2-2258-6066

Fax: +82-2-536-3589

E-mail: chungbh@catholic.ac.kr
Background/Aims: Anti-thymocyte globulin (ATG) treatment for acute T-cell mediated rejection (TCMR) can increase the risk of cytomegalovirus (CMV) infection. We aimed to evaluate the effect of valacyclovir prophylaxis against CMV infection after ATG administration as anti-rejection therapy.

Methods: We retrospectively analyzed 55 kidney transplant recipients (KTRs) receiving ATG for steroid resistant TCMR. In all KTRs, we used intravenous ganciclovir during ATG injection. In 34 KTRs treated before July 2013, we performed preemptive therapy for CMV infection after ATG therapy. They were regarded as the historic control group (CONT). After July 2013, we used valacyclovir maintenance for 1 month after ATG therapy in 21 patients (VAL). The primary outcome was the incidence of CMV infection, and the secondary outcomes were subsequent acute rejection, and graft and patient outcome.

Results: Valacyclovir prophylaxis significantly reduced the incidence of CMV infection (VAL, 9.6\% vs. CONT, 67.6\%; $p<0.001$ ), and CMV-free survival rate was higher in the VAL group compared to the CONT group $(p=0.009)$. In the VAL group, two cases of CMV infection were limited to CMV viremia, but CMV disease or syndrome $(n=3)$ was detected in the CONT group. There was no difference in graft failure (CONT, 70.5\% vs. VAL, 47.6\%; $p=0.152$ ), incidence of subsequent rejection after ATG treatment (CONT, $41.1 \%$ vs. VAL, 33.3\%; $p=0.776$ ), and graft or patient survival between the two groups. There were no major adverse events associated with valacyclovir prophylaxis.

Conclusions: In conclusion, valacyclovir prophylaxis is effective in the prevention of CMV infection after ATG treatment for steroid resistant TCMR.

Keywords: Valacyclovir; Cytomegalovirus prophylaxis; Antilymphocyte serum; Rejection therapy; Kidney transplantation

\section{INTRODUCTION}

Cytomegalovirus (CMV) infection is a representative opportunistic infection that can affect graft and patient survival in kidney transplant recipients (KTRs) [1]. Indeed, CMV infection can result in CMV disease or syndrome affecting various organs, but it can also cause opportunistic infection, acute rejection or even further secondary malignancy by dysregulation of the immune status of recipients [2]. Therefore, effective prophylaxis or early treatment for CMV infection after kidney transplantation (KT) is very important to im- 
prove allograft outcomes.

To date, various factors have been reported to be associated with the development of CMV infection, but many studies showed that the use of anti-thymocyte globulin (ATG) is one of the most important risk factors [3]. ATG is a strong lymphocyte depleting agent, and it is used as induction therapy for immunologically high risk KTRs or a secondary treatment option for steroid-resistant acute T-cell mediated rejection (TCMR). However, weakened lymphocyte activity by ATG can cause vulnerability to CMV infection in KTRs, which justifies the use of CMV prophylaxis after ATG treatment [4].

Usually, intravenous ganciclovir is used during ATG treatment for CMV prophylaxis [5]. In addition, maintenance oral ganciclovir or valganciclovir is recommended during 1 to 3 months after the completion of ATG treatment; however, the use of maintenance therapy is limited in many cases because of the high cost [6]. A recent report showed that valacyclovir is not only cost effective but it also has a similar efficacy in prevention of CMV infection in comparison with oral ganciclovir or valganciclovir in KTRs who received ATG induction therapy $[7,8]$. However, the usefulness of valacyclovir in the prevention of CMV infection in KTRs who received ATG as anti-rejection therapy has not been reported.

In this regard, the aim of this study is to evaluate the effect of maintenance valacyclovir universal prophylaxis against CMV infection after intravenous ganciclovir during ATG treatment as anti-rejection therapy for steroid resistant TCMR.

\section{METHODS}

\section{Study population}

We included a total of 55 adult KT recipients who received ATG for the treatment of steroid resistant rejection in Seoul St. Mary's Hospital between January 2010 and April 2016. TCMR was diagnosed based on Banff classification [9]. According to the anti-rejection treatment protocol of Seoul St. Mary's Hospital, TCMR was treated with 3 to 5 daily boluses of intravenous methylprednisolone (500 $\mathrm{mg}$ /day), followed by a 5 to 7 day oral steroid taper. However, when the serum creatinine level failed to decrease within 5 days, we regarded it as steroid resistant rejection and ATG was applied [10]. All recipients received ATG injection $(1.5 \mathrm{mg} / \mathrm{kg} /$ day $)$ for a week.

CMV prophylaxis protocol in KT'Rs who received ATG as anti-rejection therapy was as follows (Fig. 1): In all 55 KTRs, we used intravenous ganciclovir during ATG injection at a dose of $2.5 \mathrm{mg} / \mathrm{kg}$ twice a day, and the dose was modified according to the allograft function of each patient $[11,12]$. After the completion of ATG therapy, plasma CMV DNA real-time polymerase chain reaction (PCR) was checked on D7, 1, 3, 6, 9, and 12 months, and when the CMV copy number exceeded 2,000 copies/mL, we used intravenous ganciclovir therapy.

Before July 2013, we used preemptive therapy for CMV infection; in brief, the recipients received intravenous ganciclovir therapy only when CMV infection was detected without the use of anti-viral agent prophylaxis. These 34 KT'Rs belonged to the historic control group (CONT, $\mathrm{n}=34$ ). After July 2013, we used maintenance valacyclovir at a dosage of $2 \mathrm{~g}$ four times daily by mouth during 1 month after the completion of ATG therapy, in addition to the previous prophylaxis protocol [8]. Twenty-one patients who received this revised protocol belonged to the valacyclovir study group (VAL, $\mathrm{n}=21$ ). We compared the clinical outcomes and parameters between the CONT and VAL groups. This retrospective study was approved by the Institutional Review Board of our institution (KC140NMIo460), which waived the requirement for informed consent.

\section{Clinical and biochemical data}

We collected the baseline characteristics of the recipients, including sex and age. Data about transplantation included the number of human leukocyte antigen mismatches, pretransplant panel reactive antibody, positivity of cross match study, pretransplant CMV serostatus, and posttransplant immunosuppressant regimen. Clinical information, such as pathologic findings of rejection, time interval from transplantation to ATG treatment, duration of ATG administration, total dose of ATG, and duration of valacyclovir administration, was assessed.

\section{Efficacy of valacyclovir prophylaxis for CMV infection}

The primary outcome of this study was the incidence of CMV infection after ATG treatment for steroid resistant TCMR. CMV infection was defined as CMV viremia ( $\geq$ 

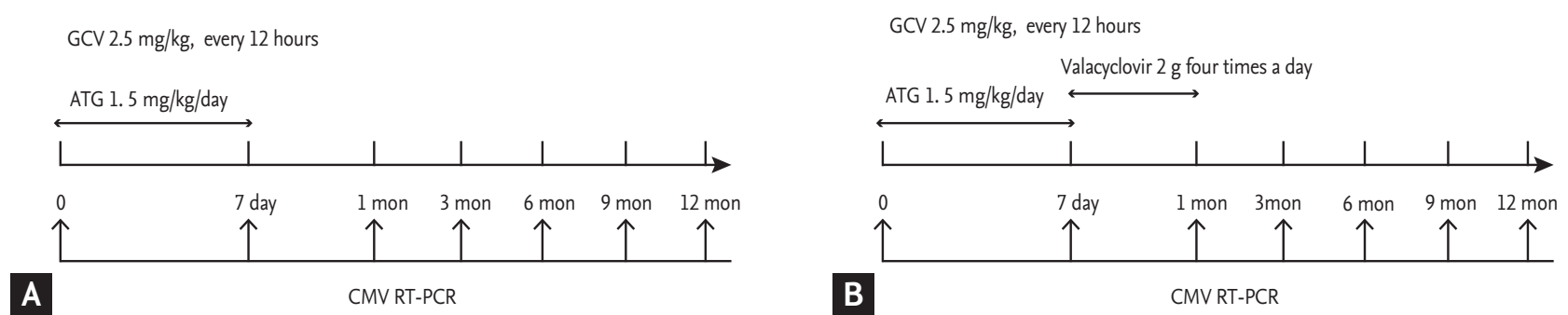

Figure 1. Cytomegalovirus (CMV) prophylaxis protocol in kidney transplant recipients (KTRs) who received anti-thymocyte globulin (ATG) as anti-rejection therapy. All KTRs were given intravenous ganciclovir (GCV) during ATG treatment (2.5 mg/kg twice a day). The control group did not receive anti-viral prophylaxis after ATG treatment, whereas valacyclovir maintenance (2 $\mathrm{g}$ four times a day by mouth) was performed until 1 month after completion of ATG treatment in the valacyclovir group. Plasma CMV DNA real-time polymerase chain reaction (RT-PCR) was checked on 7 days, 1, 3, 6, 9, and 12 months after ATG treatment.

500 copies $/ \mathrm{mL}$ ) using quantitative CMV PCR, irrespective of the symptoms. The secondary outcomes of this study were CMV disease, subsequent acute rejection, graft function assessed as estimated glomerular filtration rate by the Chronic Kidney Disease Epidemiology Collaboration formula, adverse events, death-censored allograft survival, and patient survival rate [13]. CMV disease was defined as symptomatic infection. Clinical symptoms included CMV syndrome (fever, and one or more of the following findings: constitutional symptoms, leukopenia, thrombocytopenia, or liver enzyme elevation) or tissue-invasive CMV disease [14].

\section{Statistical analysis}

Statistical analyses were performed using SPSS version 23.0 (IBM Co., Armonk, NY, USA). Data are presented as mean \pm standard deviation or counts and percentages, depending on the data type. For continuous variables, means were compared using Student $t$ test. For categorized variables, Pearson chi-square test and Fisher exact test were used. Patient, graft and CMV-free survival rates were calculated using Kaplan-Meier analysis, and we used the log-rank method to compare the survival rates between the VAL and CONT groups. All tests were two-tailed, and the results were considered statistically significant when the $p$ value was less than 0.05 .

\section{RESULTS}

\section{Comparison of baseline characteristics}

The comparison of baseline characteristics between the VAL group and the CONT group is presented in Table 1. Demographic characteristics such as patient's mean age at the time of TCMR diagnosis and sex ratio were not different between the two groups. The pretransplant immunologic status and CMV serostatus of donors and recipients, and the time interval from KT to ATG administration did not differ significantly between the two groups ( $p>0.05$, respectively) (Table 1). At the time of allograft biopsy, immune suppression regimens, pathologic findings at biopsy, allograft function and the duration or administered amount of ATG also showed no differences. The allograft function at biopsy also did not differ between the two groups. The mean duration of valacyclovir administration was 28.3 \pm 5 days in the VAL group.

\section{Comparison of the development of CMV infection}

The incidence of CMV infection was significantly lower in the VAL group $(2 / 21,9.6 \%)$ compared to the CONT group $(23 / 34,67.6 \% ; p<0.001)$ (Fig. $2 \mathrm{~A}$ ). With respect to the interval between ATG administration and CMV infection, it was significantly shorter in the CONT group ( $34.8 \pm 19$ days) than in the VAL group ( $101 \pm 33$ days, $p<$ 0.001 vs. CONT group). In the CONT group, all CMV infections occurred within the first 3 months after ATG treatment. On the other hand, in the VAL group, all cases of CMV infection occurred after 1 month. Therefore, the 1-year CMV-free survival rate was significantly higher in the VAL group compared to the control group (80\% vs. $38.6 \%, p=0.009$ ) (Fig. $2 \mathrm{~B}$ ). In the VAL group, all cases of CMV infection were limited to CMV viremia. In contrast, in the CONT group, three cases of CMV 
disease were detected; one case of CMV nephritis, proven by kidney biopsy, and two cases of CMV syndrome.

\section{Comparison of adverse events associated with an- ti-viral treatment}

The cumulative incidence of leukopenia $\left(<4.0 \times 10^{9} / \mathrm{L}\right)$

Table 1. Comparison of baseline characteristics

\begin{tabular}{|c|c|c|c|}
\hline Characteristic & $\operatorname{CONT}(\mathrm{n}=34)$ & $\operatorname{VAL}(\mathrm{n}=21)$ & $p$ value \\
\hline Recipient age, yr & $41.7 \pm 10$ & $44.8 \pm 12$ & 0.298 \\
\hline Recipient male, \% & 50 & 33 & 0.272 \\
\hline \multicolumn{4}{|l|}{ Immunologic status } \\
\hline No. of HLA mismatch & $3.6 \pm 2$ & $3.2 \pm 2$ & 0.606 \\
\hline Positive crossmatch & o & $2(9 \cdot 5)$ & 0.141 \\
\hline ABO incompatible & $3(8.8)$ & $4(19.0)$ & 0.408 \\
\hline $\mathrm{PRA}>50 \%$ & $1(2.9)$ & $1(4.8)$ & $>0.999$ \\
\hline MDRD eGFR at biopsy, $\mathrm{mL} / \mathrm{min} / 1.73 \mathrm{~m}^{2}$ & $16.5 \pm 10.9$ & $23 \cdot 3 \pm 14 \cdot 4$ & 0.074 \\
\hline Pathologic finding of rejection & & & 0.304 \\
\hline Acute TMR $1 \mathrm{~A}$ & $8(23.5)$ & $5(23.8)$ & \\
\hline Acute TMR $1 B$ & $12(35 \cdot 3)$ & $8(38.3)$ & \\
\hline Acute TMR 2A & $11(32.3)$ & $3(14.2)$ & \\
\hline Others & $3(8.8)$ & $5(23.8)$ & \\
\hline CMV seropositivity at baseline & $28(100)$ & $17(80.9)$ & 0.391 \\
\hline Time from transplantation to ATG, mon & $20.2 \pm 22$ & $33.1 \pm 35$ & 0.101 \\
\hline Duration of ATG administration, day & $6.4 \pm 2$ & $5 \cdot 9 \pm 2$ & 0.253 \\
\hline Total dose of ATG, mg & $539 \pm 216$ & $472 \pm 174$ & 0.397 \\
\hline Duration of VAL administration, day & - & $28.3 \pm 5$ & \\
\hline
\end{tabular}

Values are presented as mean $\pm \mathrm{SD}$ or number (\%).

CONT, control; VAL, valacyclovir; HLA, human leukocyte antigen; PRA, panel reactive antibody; MDRD, modification of diet in renal disease; eGFR, estimated glomerular filtration rate; TMR, T-cell mediated rejection; CMV, cytomegalovirus; ATG, anti-thymocyte globulin antibody.
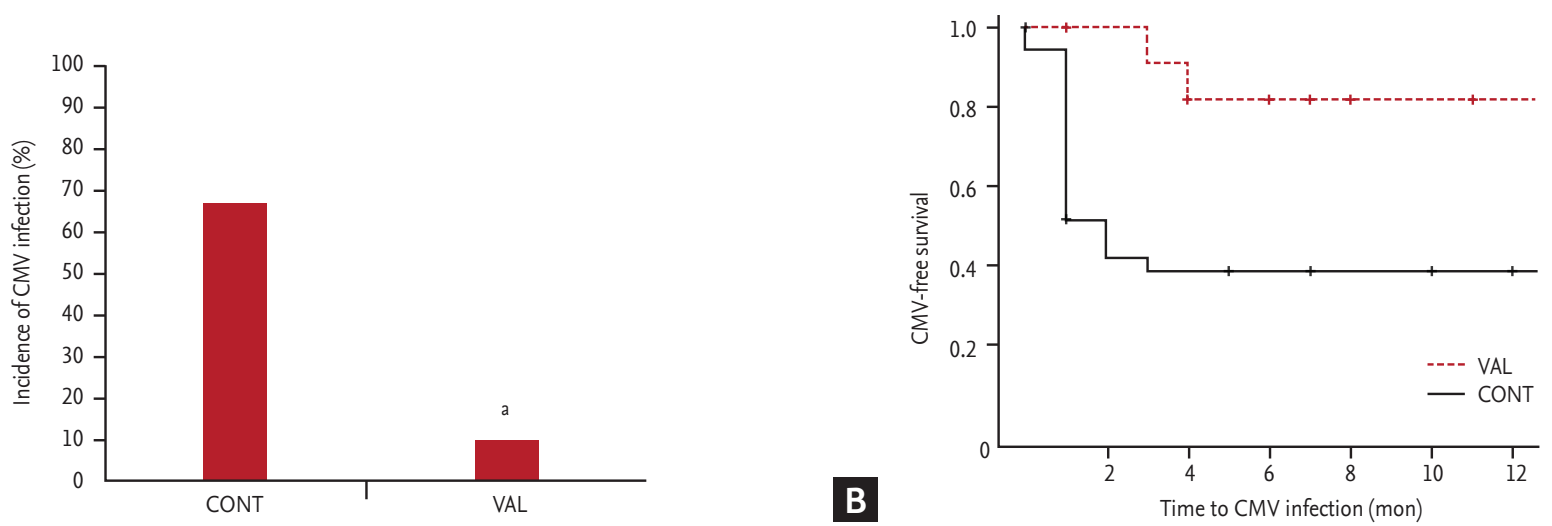

Figure 2. Comparison of the incidence of cytomegalovirus (CMV) infection and CMV-free survival rate between the control (CONT) and valacyclovir (VAL) groups. (A) Incidence of CMV infection. (B) Kaplan-Meier curves for the CMV-free survival rate. Note that the VAL group showed significantly lower incidence of CMV infection and the CMV-free survival rate was also higher compared with that in the CONT group ( $p<0.01$ for each). ${ }^{a} p<0.01$ vs. CONT. 
Ko EJ, et al. CMV prophylaxis after ATG treatment

Table 2. Comparison of adverse events

\begin{tabular}{lccc}
\hline & CONT $(\mathrm{n}=34)$ & VAL $(\mathrm{n}=21)$ & $p$ value \\
\hline Anemia & $11(32)$ & $5(23)$ & 0.556 \\
Leukopenia & $8(24)$ & $7(33)$ & 0.272 \\
Thrombocytopenia & $3(9)$ & $3(14)$ & 0.664 \\
\hline
\end{tabular}

Values are presented as number (\%).

CONT, control; VAL, valacyclovir.

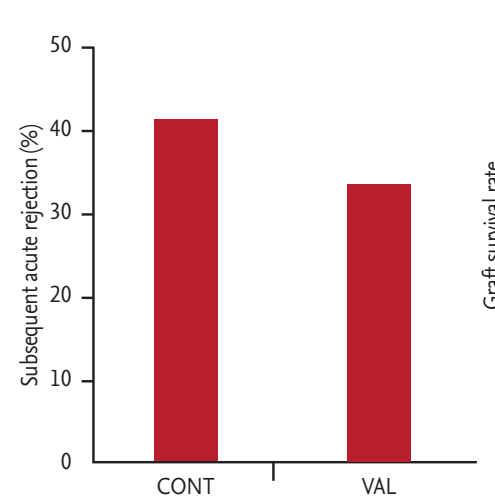

A

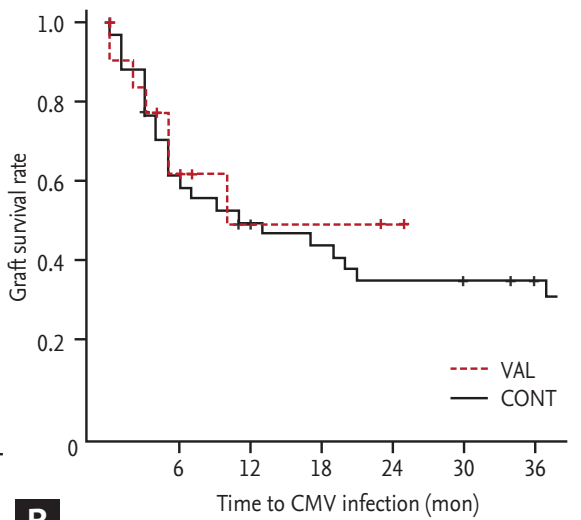

B

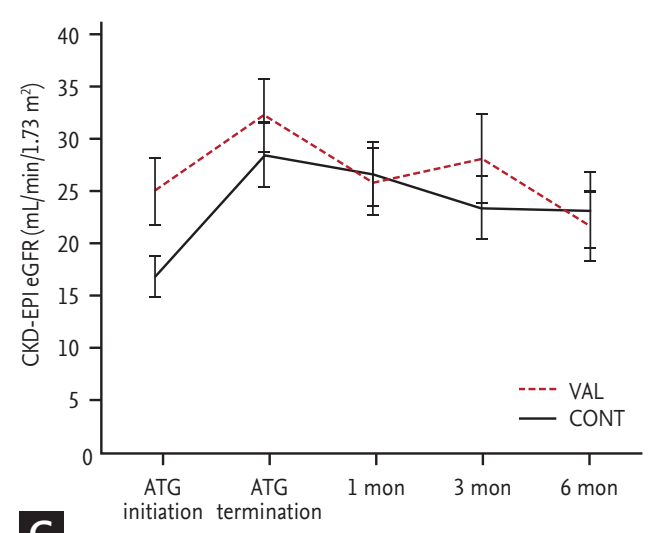

c

Figure 3. Comparison of the incidence of subsequent acute rejection, graft survival rate, and allograft function after anti-thymocyte globulin (ATG) treatment. (A) Subsequent acute rejection. (B) Kaplan-Meier curves for the graft survival rate. (C) Allograft function (CKD-EPI eGFR). Note that the incidence of subsequent acute rejection, graft survival, and allograft function after ATG treatment was not significantly different between the two groups ( $p>0.05$ for each). CONT, control; VAL, valacyclovir; CMV, cytomegalovirus; CKD-EPI, Chronic Kidney Disease Epidemiology Collaboration; eGFR, estimated glomerular filtration rate.

(CONT, $32 \%$ [11/34] vs. VAL, 23\% [5/21]; $p=0.556$ ), anemia (hemoglobin < $9 \mathrm{~g} / \mathrm{dL})(\mathrm{CONT}, 24 \%$ [8/34] vs. VAL, 33\% [7/21]; $p=0.272)$, and thrombocytopenia $(<100 \times 109 / \mathrm{L})$ (CONT, 9\% [3/34] vs. VAL, 14\% [3/21]; $p=0.664$ ), did not differ between the CONT and VAL groups (Table 2). This indicates that valacyclovir did not increase the risk of bone marrow suppression, and it had no additional effect. In six patients, valacyclovir was withheld within 1 month because of deterioration of allograft function. However, there were no clinically important side effects associated with valacyclovir such as hallucination and/or confusion.

\section{Comparison of allograft outcomes after CMV infection}

There was no significant difference in subsequent acute rejection after ATG treatment between the two groups (Fig. 3A). The incidence of subsequent acute rejection after ATG treatment was $41.1 \%(14 / 34)$ in the CONT group, whereas the incidence of subsequent acute re- jection after ATG treatment was $33.3 \%(7 / 21)$ in the VAL group $(p=0.776)$. The allograft survival rate after allograft rejection also did not differ between the two groups $(p=0.508)$ (Fig. $3 \mathrm{~B})$. In the CONT group, graft failure occurred in $70.5 \%$ of the cases $(24 / 34)$, while in the VAL group, graft failure occurred in $47.6 \%$ of the cases $(10 / 21)(p=0.152)$. Chronic rejection was the main cause of graft failure in both groups (CONT, 87.5\% [21/24] vs. VAL, $100 \%$ [10/10]; $p=0.067$ ). Allograft function showed a lower tendency in the CONT group after ATG treatment; however, the differences were not significant (Fig. 3 C). There was no patient death in the VAL group, and there were two cases of death in the CONT group. In the CONT group, one patient died of urothelial cancer of the kidney and the other patient died of atypical pneumonia. Patient survival rate showed no difference between the two groups $(p=0.480)$. 


\section{DISCUSSION}

In this study, we investigated the usefulness of valacyclovir prophylaxis against CMV infection after ATG treatment for steroid resistant TCMR. We found that the incidence of CMV infection after ATG therapy was significantly lower in the VAL group in comparison with the CONT group with similar other clinical outcomes. This suggests that valacyclovir can be recommended as a first-line prophylactic agent for patients who received ATG as anti-rejection therapy.

First, we focused on the high risk of CMV infection after ATG treatment as induction therapy or anti-rejection therapy $[4,15]$. In KTRs who received ATG as induction therapy, the incidence of CMV infection was reported to be $67 \%$, and in KTRs who received ATG therapy, it was reported to be $33 \%$, which was significantly higher than in KTRs who received only steroid pulse therapy $[2,16]$. In this study, the incidence of CMV infection in the CONT group was nearly $67.6 \%$ in spite of the use of intravenous ganciclovir during ATG treatment. This strongly suggests that maintenance therapy with an anti-viral agent after the end of ATG therapy is required, and therefore, we changed our center's CMV prophylaxis protocol in KTRs who received ATG therapy as anti-rejection therapy after July 2013 [17].

Our next concern was the choice of anti-viral agents. In case of ATG induction therapy, oral ganciclovir or valganciclovir is commonly recommended [17]. In addition, oral valacyclovir showed similar efficacy in the prevention of CMV infection in well-designed clinical trials $[7,8]$. Cost-effectiveness of valacyclovir in comparison with oral ganciclovir or valganciclovir further justified the use of this drug in these patients. Acyclovir also showed anti-CMV activity with similar cost-effectiveness as valacyclovir. However, the effectiveness and bioavailability of oral acyclovir are significantly lower than that of valacyclovir [18]. Therefore, we thought that valacyclovir could be a promising option instead of oral ganciclovir or valganciclovir, as it showed similar effectiveness and better cost-effectiveness.

Indeed, use of valacyclovir prophylaxis significantly lowered the incidence of CMV infection, and further, the infection itself was less severe in the VAL group in comparison with the CONT group in this study. There were only two episodes of CMV infections in the VAL group, which were limited to CMV viremia, the mild form of infection, and there was no clinical symptomatic CMV disease. However, there were several cases of CMV syndrome and biopsy-proven CMV nephritis in the CONT group. In addition, valacyclovir reduced the incidence of CMV infection immediately after ATG treatment, during which leukopenia is common due to ATG therapy, as shown in this study. CMV infection during this period can result in more a severe immunocompromised state, which can be associated with other secondary opportunistic infections [19-22]. This finding suggests that valacyclovir prophylaxis may prevent critical CMV infection, which may result in patient morbidity or mortality.

The final issue is the adverse effect due to maintenance anti-viral therapy. The previous study has shown that antiviral agents for CMV prophylaxis can have an adverse effect such as bone marrow suppression (anemia, leukopenia, and thrombocytopenia) or psychiatric side effect such as hallucination or confusion, which may limit the use of these maintenance drugs $[2,7,23]$. But in this study, there was no difference in bone marrow suppression events between the two groups, which suggests that these side effects are not associated with valacyclovir but with the effect of ATG administration [20-22,24-26]. In addition, there were no typical psychiatric adverse events in the VAL group. All these results suggest that proper dose modification with close monitoring can minimize the adverse effects of valacyclovir. In this study, the main reason for requiring dose reduction or discontinuation was deterioration of allograft function, and it may be because of the progression of acute rejection and not because of the use of valacyclovir.

There are some limitations to this study. At first, this was a retrospective study with a limited sample size and a single-center design. Since it was retrospective study, patient's adherence to valacyclovir could not be assessed. Also, we compared the historic control group (previous protocol) with the study group (revised protocol), which might cause selection bias. Second, we did not compare the effect of valacyclovir with that of oral ganciclovir or valganciclovir, which is generally recommended for CMV prophylaxis. Further prospective randomized-controlled trial with a large sample size is needed. 
In conclusion, given the increasing incidence of postATG CMV disease, valacyclovir could be the appropriate drug for prophylaxis for CMV disease without any concern about significant side effects after ATG treatment for steroid resistant TCMR.

\section{KEY MESSAGE}

1. Cytomegalovirus (CMV) infection affects the graft and patient survival in kidney transplant recipients.

2. Anti-thymocyte globulin (ATG) treatment for acute T-cell mediated rejection can increase the risk of CMV infection.

3. Valacyclovir prophylaxis prevents CMV infection after ATG treatment without any concern about significant side effects.

\section{Conflict of interest}

No potential conflict of interest relevant to this article was reported.

\section{Acknowledgments}

This research was supported by a grant of the Korean Health Technology R\&D Project, Ministry of Health and Welfare, Republic of Korea (HI13C1232).

\section{REFERENCES}

1. Kliem V, Fricke L, Wollbrink T, Burg M, Radermacher J, Rohde F. Improvement in long-term renal graft survival due to CMV prophylaxis with oral ganciclovir: results of a randomized clinical trial. Am J Transplant 2008;8:975-983.

2. Reischig T, Jindra P, Mares J, et al. Valacyclovir for cytomegalovirus prophylaxis reduces the risk of acute renal allograft rejection. Transplantation 2005;79:317-324.

3. Issa NC, Fishman JA. Infectious complications of antilymphocyte therapies in solid organ transplantation. Clin Infect Dis 2009;48:772-786.

4. Couzi L, Helou S, Bachelet T, et al. Preemptive therapy versus valgancyclovir prophylaxis in cytomegalovirus-positive kidney transplant recipients receiving antithymocyte globulin induction. Transplant Proc
2012;44:2809-2813

5. Said T, Nampoory MR, Johny KV, et al. Cytomegalovirus prophylaxis with ganciclovir in kidney transplant recipients receiving induction antilymphocyte antibodies. Transplant Proc 2004;36:1847-1849.

6. Hellemans R, Beutels P, Ieven M, Verpooten GA, Bosmans JL. Cost analysis in favor of a combined approach for cytomegalovirus after kidney transplantation: a single-center experience. Transpl Infect Dis 2013;15:70-78.

7. Reischig T, Opatrny Jr K, Treska V, Mares J, Jindra P, Svecova M. Prospective comparison of valacyclovir and oral ganciclovir for prevention of cytomegalovirus disease in high-risk renal transplant recipients. Kidney Blood Press Res 2005;28:218-225.

8. Reischig T, Hribova P, Jindra P, et al. Long-term outcomes of pre-emptive valganciclovir compared with valacyclovir prophylaxis for prevention of cytomegalovirus in renal transplantation. J Am Soc Nephrol 2012;23:15881597.

9. Racusen LC, Solez K, Colvin RB, et al. The Banff 97 working classification of renal allograft pathology. Kidney Int 1999;55:713-723.

10. Chung BH, Oh HJ, Piao SG, et al. Clinical significance of the ratio between $\mathrm{FOXP}_{3}$ positive regulatory $\mathrm{T}$ cell and interleukin-17 secreting cell in renal allograft biopsies with acute T-cell-mediated rejection. Immunology 2012;136:344-351.

11. Gaber AO, First MR, Tesi RJ, et al. Results of the double-blind, randomized, multicenter, phase III clinical trial of thymoglobulin versus Atgam in the treatment of acute graft rejection episodes after renal transplantation. Transplantation 1998;66:29-37.

12. Brennan DC, Flavin K, Lowell JA, et al. A randomized, double-blinded comparison of thymoglobulin versus Atgam for induction immunosuppressive therapy in adult renal transplant recipients. Transplantation 1999;67:1011-1018.

13. Levey AS, Stevens LA, Schmid CH, et al. A new equation to estimate glomerular filtration rate. Ann Intern Med 2009;150:604-612.

14. Ljungman P, Griffiths P, Paya C. Definitions of cytomegalovirus infection and disease in transplant recipients. Clin Infect Dis 2002;34:1094-1097.

15. Turgeon N, Fishman JA, Basgoz N, et al. Effect of oral acyclovir or ganciclovir therapy after preemptive intravenous ganciclovir therapy to prevent cytomegalovirus 
disease in cytomegalovirus seropositive renal and liver transplant recipients receiving antilymphocyte antibody therapy. Transplantation 1998;66:1780-1786.

16. Hibberd PL, Tolkoff-Rubin NE, Conti D, et al. Preemptive ganciclovir therapy to prevent cytomegalovirus disease in cytomegalovirus antibody-positive renal transplant recipients: a randomized controlled trial. Ann Intern Med 1995;123:18-26.

17. Kotton CN, Kumar D, Caliendo AM, et al. Updated international consensus guidelines on the management of cytomegalovirus in solid-organ transplantation. Transplantation 2013;96:333-360.

18. Ormrod D, Scott LJ, Perry CM. Valaciclovir: a review of its long term utility in the management of genital herpes simplex virus and cytomegalovirus infections. Drugs 2000;59:839-863.

19. Sagedal S, Nordal KP, Hartmann A, et al. The impact of cytomegalovirus infection and disease on rejection episodes in renal allograft recipients. Am J Transplant 2002;2:850-856.

20. Ciancio G, Gaynor JJ, Sageshima J, et al. Randomized trial of dual antibody induction therapy with steroid avoidance in renal transplantation. Transplantation 2011;92:1348-1357.

21. Kuypers DR, Malaise J, Claes K, et al. Secondary effects of immunosuppressive drugs after simultaneous pancreas-kidney transplantation. Nephrol Dial Transplant 2005;20 Suppl 2:ii33-ii39.

22. Zafrani L, Truffaut L, Kreis H, et al. Incidence, risk factors and clinical consequences of neutropenia following kidney transplantation: a retrospective study. Am J Transplant 2009;9:1816-1825.

23. Lowance D, Neumayer HH, Legendre CM, et al. Valacyclovir for the prevention of cytomegalovirus disease after renal transplantation. International Valacyclovir Cytomegalovirus Prophylaxis Transplantation Study Group. N Engl J Med 1999;340:1462-1470.

24. Grafals M, Smith B, Murakami N, et al. Immunophenotyping and efficacy of low dose ATG in non-sensitized kidney recipients undergoing early steroid withdrawal: a randomized pilot study. PLoS One 2014;9:e104408.

25. Marfo K, Akalin E, Wang C, Lu A. Clinical and economic analysis of short-course versus standard-course antithymocyte globulin (rabbit) induction therapy in deceased-donor renal transplant recipients. Am J Health Syst Pharm 2011;68:2276-2282.

26. Kute VB, Vanikar AV, Shah PR, et al. Post-renal transplant cytomegalovirus infection: study of risk factors. Transplant Proc 2012;44:706-709. 